\title{
IMPROVEMENT DRIVING CHARACTERISTICS OF ELECTRIC TRICYCLE
}

\author{
Jan Dizo, Dalibor Barta, Miroslav Blatnicky \\ University of Zilina, Slovakia \\ jan.dizo@fstroj.uniza.sk,dalibor.barta@fstroj.uniza.sk,miroslav.blatnicky@fstroj.uniza.sk
}

\begin{abstract}
The article deals with the design of a modified system for changing of the driving direction of a threewheel vehicle with electric drive. The classic solution of three-wheeled vehicles is unstable at a certain speed, which leads to dangerous tilting. The new designed steering system of such a vehicle should contribute to increasing its safety and to widening the scope for its use. The vehicle is currently designed for one person, but it is expected to increase its capacity to two persons and their luggage. It brings changes in a construction of the vehicle and results in a change in the centre of gravity. The modified solution of the vehicle steering system increases its maximum speed. The vehicle construction consists of an electric drive located above the rear axle with torque transmission to the rear wheels. The front wheel is steered. The classic steering solution is replaced by a steering wheel that facilitates steering. The article compares the stability of a vehicle with a modified steering system to the conventional solution during driving in a curve. The calculations were performed for various speeds using the multibody simulation on the virtual model in Simpack software. The solution is a part of the project supported by the Volkswagen Slovakia Foundation oriented on the support of electromobility, students and young scientists.
\end{abstract}

Keywords: tricycle, electromobility, dynamic simulation, driving characteristics.

\section{Introduction}

As a result of the sharp increase in the cost of petrol in recent years, significant pressure has been put on the automotive and recreational vehicle industries to develop more fuel efficient passenger vehicles. At present, standard four-wheeled vehicles are designed to accommodate four to six passengers comfortably with sufficient space for their luggage. This design creation limits significant improvements in the fuel efficiency of these vehicles. On the other hand, two-wheeled vehicles such as bicycles, mopeds and motorcycles are extremely fuel efficient. However, these two-wheeled vehicles are primary designed as single passenger vehicles with limited luggage compartment. In addition, they do not protect enough passengers from bad weather conditions sufficiently. This makes them unsatisfactory for the majority of the driving public. Therefore, there is a need for design of vehicles, which are intended for transportation of one or two passengers with some of the space and comfort afforded by standard four-wheeled vehicles and significantly greater fuel efficiency [1].

As one of the most effective ways for exhaust reducing [2] and fossil fuel saving electromobility is currently regarded. On the one hand, electric motors are used in various areas of passenger transport [3; 4] and, on the other hand, three-wheeled vehicles are increasingly popular. It is natural to consider electric three-wheeled vehicles (simpliciter tricycles) as means to achieve this goal.

The aim of this article is to introduce an idea and a technical solution of the front part of an electric tricycle in order to improve the driving properties, mainly its rollover stability, when it is driving in curves.

\section{Technical solution of the front wheel steering}

A technical solution relates to the front wheel fork design of an electric tricycle with two wheels on the rear axle. The goal is to improve the rollover stability of such a vehicle during driving in curves.

Nowadays, steering mechanisms of three-wheeled vehicles with two wheels on the rear axle use a classic fork for the front wheel mounted on a vehicle chassis.

In current solutions of the classic tricycle steering, the contact point between the front wheel and the road during driving in curves is changeless. Then, the arising centrifugal force is compensated by passengers tilting in the opposite direction, because the centre of gravity is near to the stability axis, which is given by the jumper lead of the front wheel and the external rear wheel in a curve.

Therefore, the requirement has occurred for the design of such front wheel suspension structure, which would ensure the rollover stability improvement of an electric tricycle and besides, the 
conditions of stability during braking have to be satisfied. The result of this effort is the new design of the front fork suspension of an electric tricycle.

The fundamental of our technical solution is the fact, that the front part of an electric tricycle contains the combination of rotating and shifting joints, which allows in addition to the rotary movement also the shifting movement in the lateral direction.

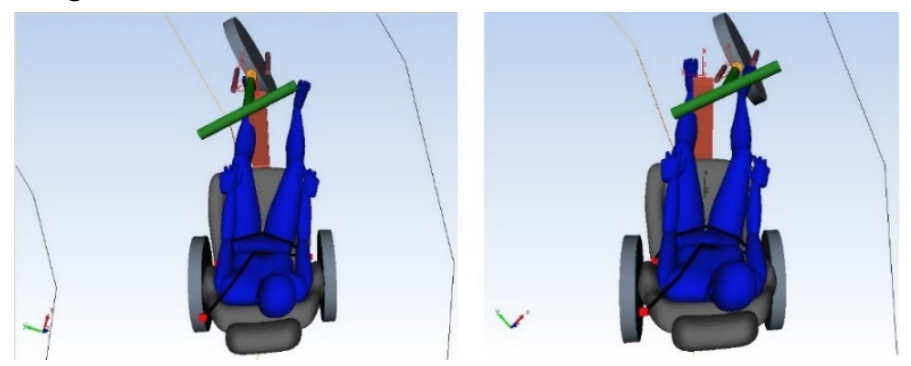

Fig. 1. Comparison of tricycle driving in curve with classic steering system (left) and with new designed steering system (right)

Comparison of the fundamentals of the difference between the classic steering system of an electric tricycle and the new designed steering system is shown in Fig. 1, where the virtual model of both is created by means of multibody Simpack package.

Advantages of the technical solution of the front wheel fork suspension of a tricycle are apparent from its effects, by which it expresses itself outwards. Effects of this technical solution are that this design allows rollover stability improvement of a tricycle, which uses steering of the motorcycle type. The introduced design is based on the front wheel shifting to the right side during driving in the left curve (Fig. 1) and contrariwise, i.e. always in the centrifugal force direction in order to improve the rollover stability. Currently, we apply our new technical solution on a three wheeled vehicle with the electric drive train, but it can be essentially used on a three wheeled vehicle with any kind of the drive train.

\section{Rollover stability during driving in a curve}

This section contains geometrical relationships governing the rollover stability due to a lateral acceleration, when a three-wheeled vehicle with two wheels on the rear axle is driving in a curve. This lateral acceleration is assumed to act perpendicular to the centre line of the vehicle. There are compared two technical solutions, the classic tricycle and the new design of a front wheel fork, which allows except for the inevitable rotation also the lateral shifting of the front wheel $[1 ; 5 ; 6]$.

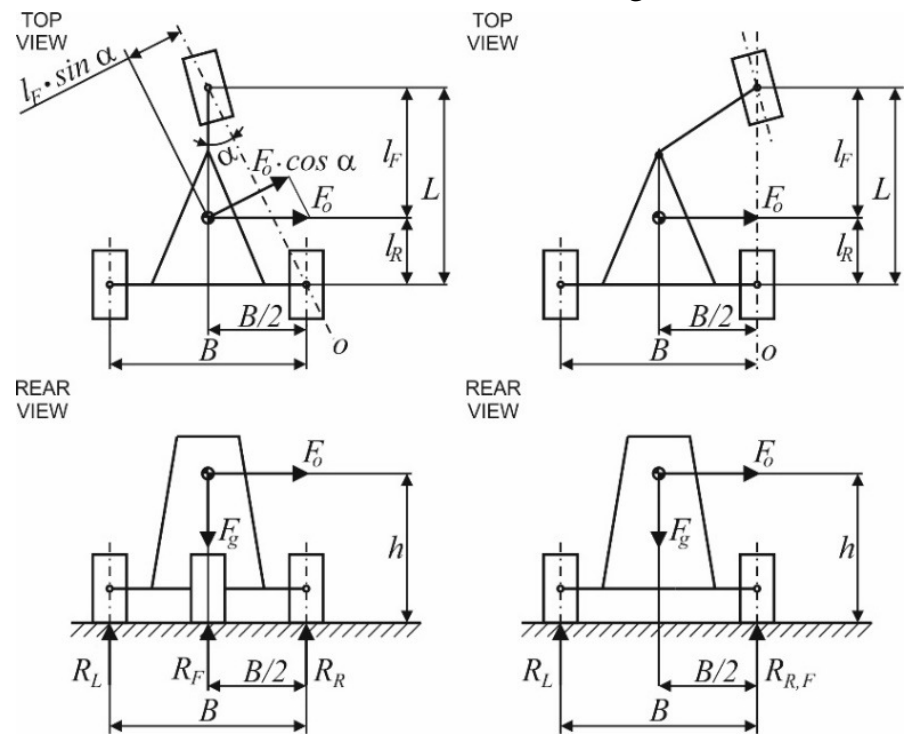

Fig. 2. Scheme of classic tricycle (left) and scheme of new designed tricycle (right)

Fig. 2 illustrates the top and rear views of a three-wheeled vehicle with two wheels on the rear axle, whereby the classic tricycle is shown on the left and the new designed tricycle is shown on the 
right, respectively. In Fig. 2, the $O$ axis represents the axis of tipping. To ensure rollover stability, a negative clockwise moment around the $O$ axis, as viewed from the rear view of the vehicle, must exist [1].

Let us consider the classic three-wheeled vehicle model shown in Fig. 2, left. Summing moments about the $O$ axis gives the equation:

$$
\sum_{i} M_{i O} \leq 0 \Rightarrow F_{o} \cdot \cos \alpha \cdot h-F_{g} \cdot l_{F} \cdot \sin \alpha \leq 0,
$$

where $F_{o}-$ force causes lateral acceleration - a centrifugal force, N;

$h$ - height of the centre of gravity of a vehicle, m;

$l_{F}$ - distance of the centre of gravity from the front wheel, $\mathrm{m}$;

$\alpha-$ angle given by main dimensions of a vehicle, ${ }^{\circ}$.

The equation (1) we can rewrite as:

$$
\frac{a_{o}}{g} \leq \frac{l_{F}}{h} \cdot \tan \alpha,
$$

where $g$-gravity acceleration, $\mathrm{m} \cdot \mathrm{s}^{-2}$,

and it represents the rollover stability condition for the classic tricycle.

As the $\alpha$ angle is a function of the geometry of a vehicle, it can be shown, that:

$$
\tan \alpha=\frac{B}{2 \cdot L}
$$

where $B$ - rear wheels gauge, $\mathrm{m}$;

$L-$ axle base, $\mathrm{m}$.

Substituting eq. (3) into eq. (2) yields the following rollover stability condition for the threewheeled vehicle with two rear wheels:

$$
\frac{a_{o}}{g} \leq \frac{B}{2 \cdot h} \cdot \frac{l_{F}}{h} .
$$

Now, let us consider the new designed tricycle, which has the new technical solution of the steering system enabling the lateral shifting of the front wheel (Fig. 2, right). Summing moments about the tipping axis $O$ gives:

$$
\sum_{i} M_{i O} \leq 0 \Rightarrow F_{o} \cdot h-F_{g} \cdot \frac{B}{2} \leq 0 .
$$

It can be rewritten as:

$$
\frac{a_{o}}{g} \leq \frac{B}{2 \cdot h}
$$

To obtain some physical feeling for the conditions that might cause the vehicle overturning we consider that each of the above considered vehicles is negotiating a curve of the radius $R$. The corresponding lateral acceleration for this case can be expressed in terms of the vehicle forward speed $v$ and the curve radius $R$. We go out from the formula:

$$
a_{o}=\frac{v^{2}}{R},
$$

where $a_{o}$ - lateral acceleration, $\mathrm{m} \cdot \mathrm{s}^{-2}$;

$v$ - vehicle speed, $\mathrm{m} \cdot \mathrm{s}^{-1}$;

$R$ - radius of a curve, $\mathrm{m}$;

If we now substitute eq. (7) into the rollover stability equations for the classic tricycle (eq. (4)) and also for the new designed tricycle yields (eq. (6)) the equation for the maximal vehicle's forward speed $v_{\max }$, at which rollover occurs [1]. Then, equations for the rollover speed are given by: 


$$
v_{\max } \leq \sqrt{R \cdot g \cdot \frac{l_{F}}{L} \cdot \frac{B}{2 \cdot h}}
$$

for a tricycle with the classic steering system and by

$$
v_{\max } \leq \sqrt{R \cdot g \cdot \frac{B}{2 \cdot h}}
$$

for a tricycle with the new designed steering system. In principle, the eq. (9) gives the relationship for the maximal forward speed determination valid for a four-wheeled vehicle.

From the derived equations (8) and (9) we can identify that $l_{F}$ is always less than $L$, the speed at which rollover for the classic tricycle will always be lower than for the corresponding new designed tricycle.

Let us have a look at Fig. 2 once more. We can see that our technical solution leads to the steering system, which would allow in the limited case such a configuration of the front wheel fork, in which the front wheel would reach the position similar (or the same) to the four-wheeled vehicle and thus also to the rollover stability improvement of the tricycle during driving in a curve.

\section{Calculations and simulations of tricycle driving properties}

In this part of the article a virtual model of two versions of a tricycle are presented - with the classic steering system and with the new designed steering system. The virtual model was created in Simpack software in order to evaluate and to compare the dynamic properties and driving characteristics of these vehicles. Further, we have also compared the analytical approach with numerical calculations of the same selected parameters. The mentioned software enables modelling and analysing of the dynamics of road vehicles, rail vehicles $[7 ; 8]$ and similar transport means ranging from trams $[9 ; 10]$ to high-speed trains. It includes various tire/road contact models and wheel/rail contact models [11], which represent an inseparable and a quite difficult modelling problem [12-14].

In order to achieve such a virtual model, which represents as faithfully as possible the reality, we have created CAD models of individual parts of both tricycle versions. Based on these, we have found the mass and inertia parameters, which were input subsequently into MBS models. Of course, moreover, the model of a driver was also included. When we have already set up MBS models, we were able to find out the total mass and inertia parameters, and the position of the total centre of gravity needed for analytical calculations.

As we wanted to investigate, how our new designed steering system can improve the tricycle rollover stability, we have chosen the driving manoeuvre in an opposing $\mathrm{S}$ curve with relatively small radius of $R=1.5 \mathrm{~m}$. Then, we performed several simulations at various speeds and we detected, when the rollover stability is breached. As the criteria of this, we have determined the contact force between the rear internal wheel in a curve and the road. The limited case is, when the contact force equals zero.

As we can mentioned above, the rollover stability criteria for both the classic steering system and the new designed steering system does not depend on the mass of the vehicle, but only on the centre of gravity position (eqs. (8), (9)) and the same construction parameters of vehicles. If we consider that the required parameters resulting from the vehicle design are $L=1.317 \mathrm{~m}$ and $B=0.727 \mathrm{~m}$ and the position of the centre of gravity is given by the two parameters, i.e. $h=0.43 \mathrm{~m}$ and $l_{F}=0.935 \mathrm{~m}$, then from eq. (1) we can calculate the maximum speed of the tricycle with the classic steering system for the limited case of the rollover stability:

$$
v_{\max } \leq \sqrt{1.5 \cdot 9.81 \cdot \frac{0.935}{1.317} \cdot \frac{0.727}{2 \cdot 0.43}} \Rightarrow v_{\max } \leq 2.972\left[\mathrm{~m} \cdot \mathrm{s}^{-1}\right]=10.699\left[\mathrm{~km} \cdot \mathrm{h}^{-1}\right]
$$

The results from the numerical analyses are shown in Fig. 3 and Fig 4. These figures contain results for the classic steering system (Fig. 3) and also for the new designed steering system (Fig. 4). There are plotted waveforms of values of total vertical wheel forces in the contact of the rear internal wheel and the road. There are also imaged the number values of this parameter. 
If we compare the result from the analytical calculation (eq. (10)) with the result from the numerical calculation (Fig. 3, green curve), we can see that the limited value of the tricycle speed of the rollover stability is really properly found out. The little difference is caused by the fact that during tricycle curving the passenger shifts in the lateral acceleration direction and thus the centre of gravity also shifts a bit in this direction.

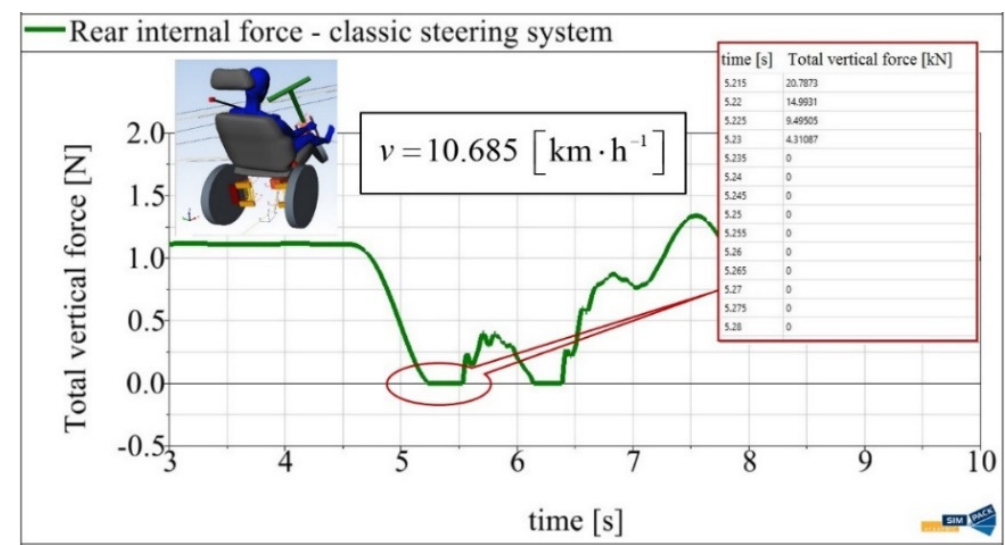

Fig. 3. Results of numerical analyses of electric tricycle with classic steering system

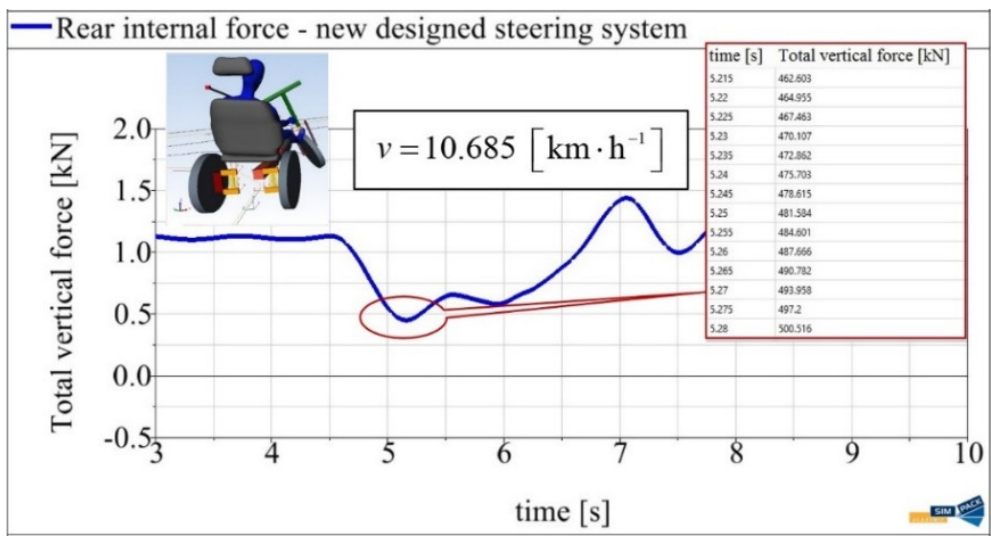

Fig. 4. Results of numerical analyses of electric tricycle with new designed steering system

Further, from the results of numeral analysis of the tricycle with the new designed steering system we can observe (Fig. 4, blue curve) that the designed technical solution improves the rollover stability of such a tricycle. It results from the fact that the rear internal wheel during driving in the curve does not lose contact with the road at all, and due to the parameters of the tricycle and driving conditions, contact forces on the rear internal wheel have sufficient values throughout the time of driving in the curve to ensure satisfactory rollover stability. It is necessary to note that in our calculation (both analytical and numerical) we have focused purely on the comparison of the original steering system with the new designed steering system and we have not considered some other phenomena, which can influence the rollover stability of the vehicle, e.g., effects of a drive train, etc. and even which can cause different effects on the left and right turning.

Based on the performed calculation, both analytical and numerical, we have found that our designed steering system is able to improve the rollover stability of a tricycle. Implementation of the solution for real use and operation still faces same problems. Therefore, our future research in this field will be focused on this prototype improvement and on the development of this technical solution in such a manner, which will be acceptable, on the one hand, from the utility point of view and, on the other hand, from the point of view of its simple application for the common use.

\section{Conclusions}

In this article a new idea of the technical solution of the new designed steering system is presented. As electromobility is currently one of the key factors of reducing the consumption of fossil natural resources, the design is intended for use on an electric three-wheeled vehicle. Our steering system is designed in order to improve the driving characteristics, particularly the vehicle rollover 
stability in curves, which is closely related to the passenger's safety. As the essential advantage of this solution, there can be considered the fact that it is purely based on the mechanical principle.

From calculation we found out that the new designed steering system really allows to improve the rollover stability of a tricycle. It was proved based on the comparison of analyses of driving these tricycles in a curve at the same driving conditions (speed, curve radius). For implementation of the improving steering system it is necessary to perform some technical modifications in such way that the operation of the electric tricycle with the new steering system will be safe, comfortable and effective.

\section{Acknowledgements}

This work was supported by the Cultural and Educational Grant Agency of the Ministry of Education of the Slovak Republic in the project No. KEGA 007ŽU-4/2017: Modernization of the vehicle and engines study programs.

This work was supported by the Grant program of the Volkswagen Slovakia Foundation in the project: E-3kolka.

\section{References}

[1] Huston J.C., Graves B.J., Johnson D.B. Three wheeled vehicle dynamics. SAE Technical Papers, 1982, pp. 45-58.

[2] Sarkan B., Stopka O., Gnap J., Caban J. Investigation of exhaust emissions of vehicles with the spark ignition engine within emission control. Procedia Engineering, Vol. 187, 2017, pp. 775-782.

[3] Skrucany T., Kendra M., Skorupa M., Grencik J., Figlus T. Comparison of chosen environmental aspects in individual road transport and railway passenger transport. Procedia Engineering, Vol. 192, 2017, pp. 806-811.

[4] Bureika G., Steisunas S. Complex evaluation of electric rail transport implementation in Vilnius city. Transport Problems, Vol. 11, No. 1, 2016, pp. 49-60.

[5] Jubin Antony J., Jaybal K. Rollover dynamics of a narrow tilting three-wheeled vehicle. MATEC Web of Conferences, Vol. 51, 2016, 8 p.

[6] Barote J., Darling J., Plummer A. Lateral dynamics simulation of a three-wheeled tilting vehicle. Proceedings of the Institution of mechanical Engineers, Part D: Journal of Automobile Engineering, Vol. 229, No. 3, 2015, pp. 342-356.

[7] Hauser V., Nozhenko O., Kravchenko K., Loulova M., Gerlici J., Lack T. Proposal of a mechanism for setting bogie wheelsets to radial position while riding along track curve. Manufacturing Technology, Vol. 17, No. 2, 2017, pp. 186-192.

[8] Melnik R., Koziak S. Rail vehicle suspension condition monitoring - approach and implementation. Journal of Vibroengineering, Vol. 19, No. 1, 2017, pp. 487-501.

[9] Hauser V., Nozhenko O., Kravchenko K., Loulova M., Gerlici J., Lack T. Proposal of a steering mechanism for tram bogie with three axle boxes. Procedia Engineering, Vol. 192, 2017, pp. 289-294.

[10] Hauser V., Nozhenko O., Kravchenko K., Loulova M., Gerlici J., Lack T. Impact of three axle boxes bogie to the tram behaviour when passing curved track. Procedia Engineering, Vol. 192, 2017, pp. 295-300.

[11] Gerlici J., Lack T. Contact geometry influence on the rail/wheel surface stress distribution. Procedia Engineering, Vol. 2, No. 1, 2010, pp. 2249-2257.

[12] Gerlici J., Lack T. Modified HHT method for vehicle vibration analysis in time domain utilisation. Applied mechanics and Materials, Vol. 486, 2014, pp. 396-405.

[13] Lack T., Gerlici J. A modified strip method to speed up the calculation of normal stress between wheel and rail. Applied Mechanics and Materials, Vol. 486, 2014, pp. 359-370.

[14] Lack T., Gerlici J. A modified strip method to speed up the tangential stress between wheel and rail calculation. Applied Mechanics and Materials, Vol. 486, 2014, pp. 371-378. 